\title{
Gene sequence analysis and screening of feature genes in spinal cord injury
}

\author{
ZONGDE YANG $^{1 *}$, XIN CHEN $^{2 *}$, REN LIU $^{3 *}$, CHUANFENG WANG $^{1}$, \\ YINCHUAN ZHAO ${ }^{1}$, ZHICAI SHI ${ }^{1}$ and MING LI ${ }^{1}$
}

\begin{abstract}
Departments of ${ }^{1}$ Spine Surgery and ${ }^{2}$ Neurosurgery, Changhai Hospital, Second Military Medical University, Shanghai 200433; ${ }^{3}$ Department of Anesthesia, Fuzhou General Hospital of Nanjing Military Command Affiliated to Fujian Medical University, Fuzhou, Fujian 350025, P.R. China
\end{abstract}

Received April 3, 2014; Accepted December 19, 2014

DOI: $10.3892 / \mathrm{mmr} .2015 .3220$

\begin{abstract}
The aim of the present study was to screen for feature genes associated with spinal cord injury (SCI), in order to identify the underlying pathogenic mechanisms. Differentially expressed genes were screened for using pre-processing data. Kyoto Encyclopedia of Genes and Genomes pathway enrichment analysis was performed to analyze and identify the genes involved in pathways associated with SCI. Subsequently, Gene Ontology enrichment analysis and Uniprot tissue analysis were used to screen out genes specifically expressed in spinal cord tissue. In addition, a protein-protein interaction network was used to demonstrate possible associations among SCI-associated feature genes. Finally, a link was identified between feature genes and SCI by analyzing protein domains in coding areas of the three feature genes. The cytochrome $c$ oxidase subunit Va, adenosine triphosphate (ATP) synthase, H+ transporting, mitochondrial F1 complex, $\alpha$ subunit 1 and cardiac muscle and mitochondrial $\beta$-F1-ATPase may be downregulated in SCI, resulting in destruction of the mitochondrial electron transport chain and membrane-bound enzyme complexes/ ion transporters, thus, affecting the normal function of nerves. The three screened feature genes have the potential to become candidate target molecules to monitor, diagnose and treat SCI and may be beneficial for the early diagnosis and therapeutic control of the condition.
\end{abstract}

Correspondence to: Dr Zhicai Shi or Dr Ming Li, Department of Spine Surgery, Changhai Hospital, Second Military Medical University, 168 Changhai Road, Shanghai 200433, P.R. China

E-mail: zhicaishizch@163.com

E-mail: minglimml@126.com

${ }^{*}$ Contributed equally

Key words: spinal cord injury, pathway analysis, feature genes, protein-protein interaction network

\section{Introduction}

The spinal cord is a part of the central nervous system in humans and other vertebrates (1). Spinal cord injury (SCI) is damage to the spinal cord, which is categorized according to the extent of loss of function, loss of sensation and the inability of the individual to stand or walk (2). It often results in confinement to a wheelchair and a lifetime of medical comorbidity (3). SCI may result from serious accidents, including road traffic accidents or sports injuries, but may also occur accompanying serious diseases, including developmental disorders, neurodegenerative diseases or demyelinating diseases. Multiple sclerosis, transverse myelitis resulting from stroke or inflammation and vascular malformations can all result in severe consequences and high-disability due to SCI (4).

Several genes and signaling pathways are involved in spinal cord injury (5). Expression of nerve growth factor, brain-derived neurotrophic factor (BDNF), neurotrophin-3 (NT-3), p75 low-affinity nerve growth factor receptor, transforming tyrosine kinase B and interleukin (IL)-6 have been reported to increase in non-neuronal cells and neuronal cells, suggesting that these molecules may be involved in promoting axonal sprouting in the injured spinal cord (6). Furthermore, it has been demonstrated that upregulation of IL-1 $\beta$, BDNF and NT-3 in the injured spinal cord is attenuated by treatment with high-dose glucocorticoids, with the suggestion that the downregulation of BDNF and NT-3 may be disadvantageous to the survival and axonal sprouting of spinal neurons (7). As for the pathways involved, a previous study revealed that the Rho signaling pathway may be a potential target for therapeutic interventions following SCI (8). In addition, apoptosis signal-regulating kinase 1 and stress-activated mitogen-activated protein kinase pathways, have also been reported to be involved in the transmission of apoptotic signals following SCI (9). However, identification and evaluation of specific and associated genes of SCI, which assist in the clinical diagnosis and treatment of SCI, remain to be elucidated.

In the present study, bioinformatics methods were used to assess the abnormal gene expression in SCI to determine the associated feature genes. Critical genes were screened using expression profiling microarray data. Pathway analysis and protein-protein interaction (PPI) network analysis were performed on the proteins involved in SCI to investigate their 
function. The aim of the present study was to explore the molecular mechanisms of SCI and identify potential therapeutic target genes for the treatment of SCI.

\section{Materials and methods}

Data preprocessing and differential expression analysis. The transcription profile of GSE2599 was downloaded from the Gene Expression Omnibus database (http://www.ncbi.nlm.nih. gov/geo/), which was based on the Affymetrix Rat Genome U34 array (Affymetrix, Santa Clara, CA, USA) and deposited by Aimone et al (10). A total of six tissue specimens were available for further analysis, including three SCI samples, obtained from female Fischer 344 rats (165-200 g) 35 days after SCI, and three normal tissues, as described in the original experiment (10). The annotation information of all probe sets was provided by Affymetrix, where the raw data (CEL) file was downloaded.

Initially, the probe-level data in the CEL files were converted into expression measures. For each sample, the expression values of all the probes for a particular gene were reduced to a single value by calculating the average expression value. Probes corresponding to more than one gene were discarded. Subsequently, the data with the low signal strength was missing data and the missing data was imputed using the K-nearest neighbor averaging (KNN) method (11) and the complete data were standardized (12). The Samr package in R language (13) was used to identify differentially expressed genes (DEGs) between three samples in the control group (normal specimen) and three samples in the experimental group (samples with SCI). In order to circumvent the multi-test problem, which may induce an excess of false positive results, the Benjamini-Hochberg procedure (14) was used to adjust the raw P-values into false discovery rate (FDR). FDR $<0.05$ and $\mid \log F C l>1.5$ were used as the cut-off criteria for DEG identification.

Kyoto Encyclopedia of Genes and Genomes (KEGG) pathway and Gene Ontology $(G O)$ enrichment analysis. Based on the deficiency of individual gene analysis, gene set enrichment analysis evaluates differential expression patterns of gene groups to distinguish whether their biological functions and characteristics differ (15). In the present study, the P-value indicated the probability that a gene was randomly endowed a GO function and it was usually used as the criterion for assigning a certain function to a module. A lower P-value increased the probability that the function of a module had not been assigned randomly, but with the purpose of performing a certain biological function, and it has important biological significance (16). The Database for Annotation, Visualization, and Integrated Discovery (DAVID) (17) bioinformatics resource consists of an integrated biological knowledge base and analytical tools aimed at systematically extracting biological meaning from lists of genes or proteins (18). The functional enrichment analysis for the screened DEGs was performed using DAVID, and $\mathrm{FDR}<0.01$ and $\mathrm{P}<0.05$ were selected as the cut-off criteria. Subsequently, KEGG pathway analysis was performed on the upregulated and downregulated genes, obtained using DAVID, to screen for disease-associated pathways.
Uniprot (UP) tissue analysis. GO analysis has become a commonly used approach for functional investigations of large-scale genomic or transcriptomic data (19). DAVID, a high-throughput and integrated data-mining environment, analyzes gene lists derived from high-throughput genomic experiments (20).

In the present study, UP tissue analysis was performed on DEGs in disease-associated metabolic pathways to identify the genes associated with spinal cord tissue. Therefore, the abnormally expressed genes in the injured spinal cord 35 days after injury were selected to distinguish these genes from those, which were expressed not solely in injured spinal cord.

Construction of the PPI network. PPI analysis was performed on the DEGs using Search Tool for the Retrieval of Interacting Genes/Proteins (STRING; http://www.string-db.org/) online database (21).Combined_score was used to measure the strength of the interaction of protein pairs and only the interaction with combined_score $>0.4$ was selected as significant. Subsequently, critical genes, which exhibited $>45$ interactions with other genes, were selected. The feature genes associated with SCI were identified by comparing the critical genes with the DEGs 35 days after SCI. Finally, the PPI network was constructed using Cytoscape software (http://cytoscape.org/) (22,23), based on the STRING database, to determine the association between feature genes and the interacting genes, which may trigger SCI.

Protein domain analysis of specific genes. Coding area prediction of the critical genes associated with SCI was performed using the GENSCAN (http://genes.mit.edu/GENSCAN.html) online software programme (24). Subsequently, the Pfam (25) database was used to examine the protein domain for further protein domain analysis.

\section{Results}

Data pre-processing and screening for DEGs. The results of data pre-processing are shown in Fig. 1. Following data pre-processing, the median was almost identical between the samples, indicating good normalization and that the data was suitable for further analysis. A total of 929 DEGs were screened for, including 339 upregulated genes and 590 downregulated genes (Fig. 2).

KEGG pathway enrichment analysis. As shown in Table I, the pathways associated with SCI included Huntington's disease (rno 05016), Parkinson's disease (rno 05012) and Alzheimer's disease (rno 05010). A total of 39 mutual genes were identified between these pathways and all of these genes were downregulated, as shown in Table II.

GO enrichment analysis. DAVID was used to identify over-represented GO categories among the genes (Table II) and $\mathrm{P}<0.05$ and FDR $<0.01$ were selected as thresholds. The most markedly enriched five terms among these genes in the PPI network were all associated with the chondriosome (Table III). GO terms associated with the mitochondria, which were enriched in the network, included the 'mitochondrial inner membrane', 'organelle inner membrane' and 'mitochondrial envelope'. 
Table I. Kyoto Encyclopedia of Genes and Genomes pathway enrichment analysis.

\begin{tabular}{lccc}
\hline Term & P-value & False discovery rate & Up/downregulated \\
\hline rno03010: Ribosome & $3.22 \mathrm{E}-12$ & $3.79 \mathrm{E}-09$ & Upregulated \\
rno04612: Antigen processing and presentation & $4.13 \mathrm{E}-08$ & $4.85 \mathrm{E}-05$ & Upregulated \\
rno00190: Oxidative phosphorylation & $2.50 \mathrm{E}-23$ & $2.98 \mathrm{E}-20$ & Downregulated \\
rno05016: Huntington's disease & $1.94 \mathrm{E}-21$ & $2.31 \mathrm{E}-18$ & Downregulated \\
rno05012: Parkinson's disease & $9.73 \mathrm{E}-21$ & $1.16 \mathrm{E}-17$ & Downregulated \\
rno05010: Alzheimer's disease & $7.00 \mathrm{E}-20$ & $8.33 \mathrm{E}-17$ & Downregulated \\
& & & \\
\hline
\end{tabular}

Table II. Downregulated genes in Huntington's disease, Parkinson's disease and Alzheimer's disease.

\begin{tabular}{llll}
\hline Uqcrc2 & Atp5o & Ndufb5 & Cox7a2 \\
Atp5d & Atp5j & Ndufb6 & Ndufa3 \\
Atp5b & Ndufb10 & Ndufb8 & Ndufa8 \\
Cyc1 & Cycs & Ndufb9 & Ndufa6 \\
Ndufab1 & Ndufc2 & Cox7b & Sdha \\
Cox5a & Cox4i1 & Atp5g1 & Ndufv2 \\
Uqcrfs 1 & Uqcr & Ndufb2 & Cox6a1 \\
Cox5b & Atp5c1 & Ndufa4 & Atp5a1 \\
Ndufs7 & Ndufb3 & Loc688869 & Ndufa5 \\
Ndufs5 & Ndufb4 & &
\end{tabular}

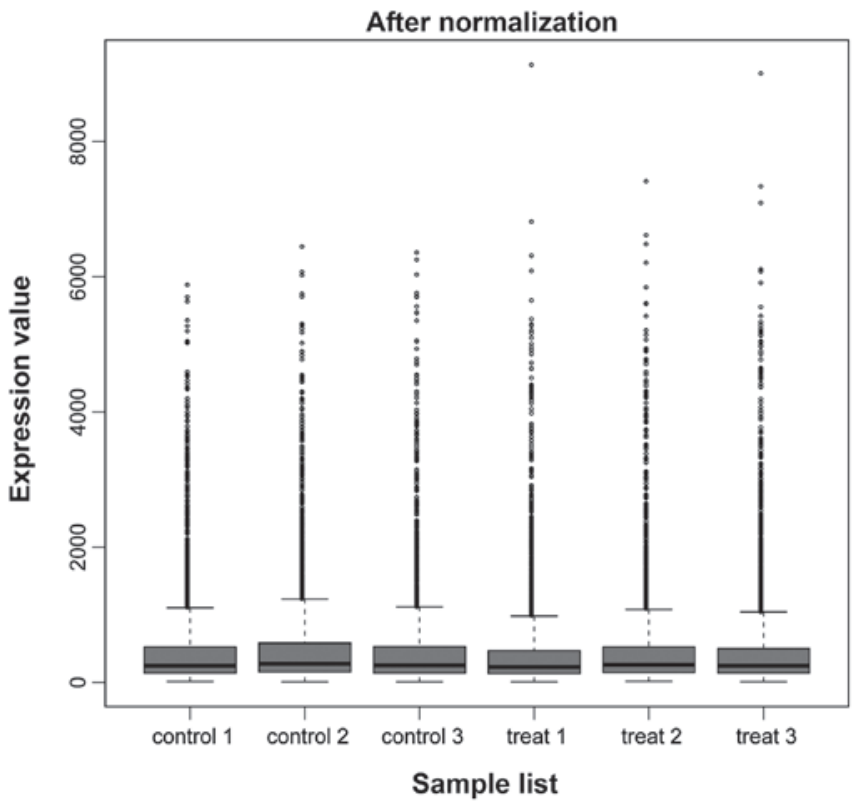

Figure 1. Box pattern of expression data following normalization. The horizontal axis indicates different samples and the vertical axis indicates the expression value. The black line within each box indicates the median of each group of data, which revealed the extent of normalization of the data. The black lines were almost on the same straight line, indicating a high level of normalization.

Identification offeature genes in SCI. Downregulated genes, which were abnormally expressed following SCI, were also involved in several known nerve disease pathways, including Huntington's disease (rno 05016), Parkinson's disease

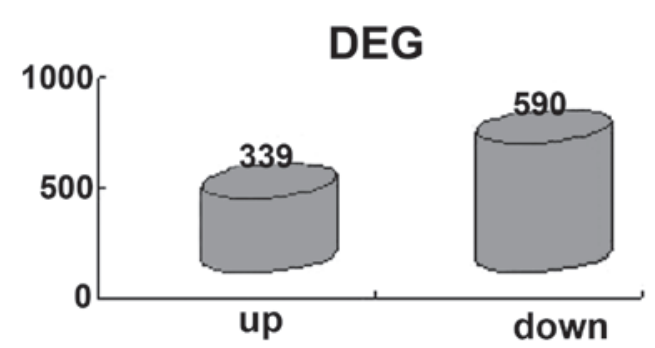

Figure 2. Number of differentially expressed genes (DEGs) that were upregulated (up; 36.5\%) and downregulated (down; 62.5\%) in spinal cord injury tissue compared with that of normal tissue.

(rno 5012) and Alzheimer's disease (rno 05010) KEGG pathways. Combined with the annotation information of spinal-cord-specific expressed genes from the Uniprot database, a candidate set of SCI-associated feature genes was obtained, including Sdha, Uqcrc2, Ndufa5, Atp5b, Atp5a1 and Cox 5 a.

PPI network analysis. Feature genes were obtained by further analysis of abnormally expressed genes in the injured spinal cord. Subsequently, a PPI network was constructed, as shown in Fig. 3, which revealed that Atp5b, Atp5a1 and Cox5a, all downregulated genes, were closely associated with the SCI when examined 35 days after the SCI. Additionally, the majority of genes interacting with these three genes were also downregulated.

Protein-domain analysis. The protein domain in the coding area of the Atp5b, Atp5a1 and Cox5a feature genes, among 
Table III. The five most enriched genes in Gene Ontology enrichment analysis.

\begin{tabular}{lllr}
\hline Category & \multicolumn{1}{c}{ GO term } & P-value & FDR \\
\hline CC & 0005743: Mitochondrial inner membrane & 4.40 E-53 & 4.14 E-50 \\
CC & 0019866: Organelle inner membrane & 4.34 E-52 & 4.09 E-49 \\
CC & 0031966: Mitochondrial membrane & 3.90 E-49 & 3.67 E-46 \\
CC & 0005740: Mitochondrial envelope & 4.49 E-48 & 4.22 E-45 \\
CC & 00444429: Mitochondrial part & 1.43 E-43 & 1.34 E-40
\end{tabular}

GO, Gene Ontology; CC, cellular component; FDR, false discovery rate.

Table IV. Protein domain in coding areas of feature genes associated with diseases 35 days after spinal cord injury.

\begin{tabular}{lllr}
\hline Gene & \multicolumn{1}{c}{ Family } & \multicolumn{1}{c}{ Description } & P-value \\
\hline Cox5a & COX5A & Cytochrome $c$ oxidase subunit Va & 2.50 E-58 \\
Atp5a1 & ATP-synt_ab_N & ATP synthase $\alpha / \beta$ family, $\beta$-barrel domain & 3.60 E-17 \\
Atp5b & ATP-synt_ab & ATP synthase $\alpha / \beta$ family, nucleotide-binding domain & 2.50 E-72 \\
& ATP-synt_ab_C & ATP synthase $\alpha / \beta$ chain, C terminal domain & 1.50 E-26
\end{tabular}

ATP, adenosine triphosphate.

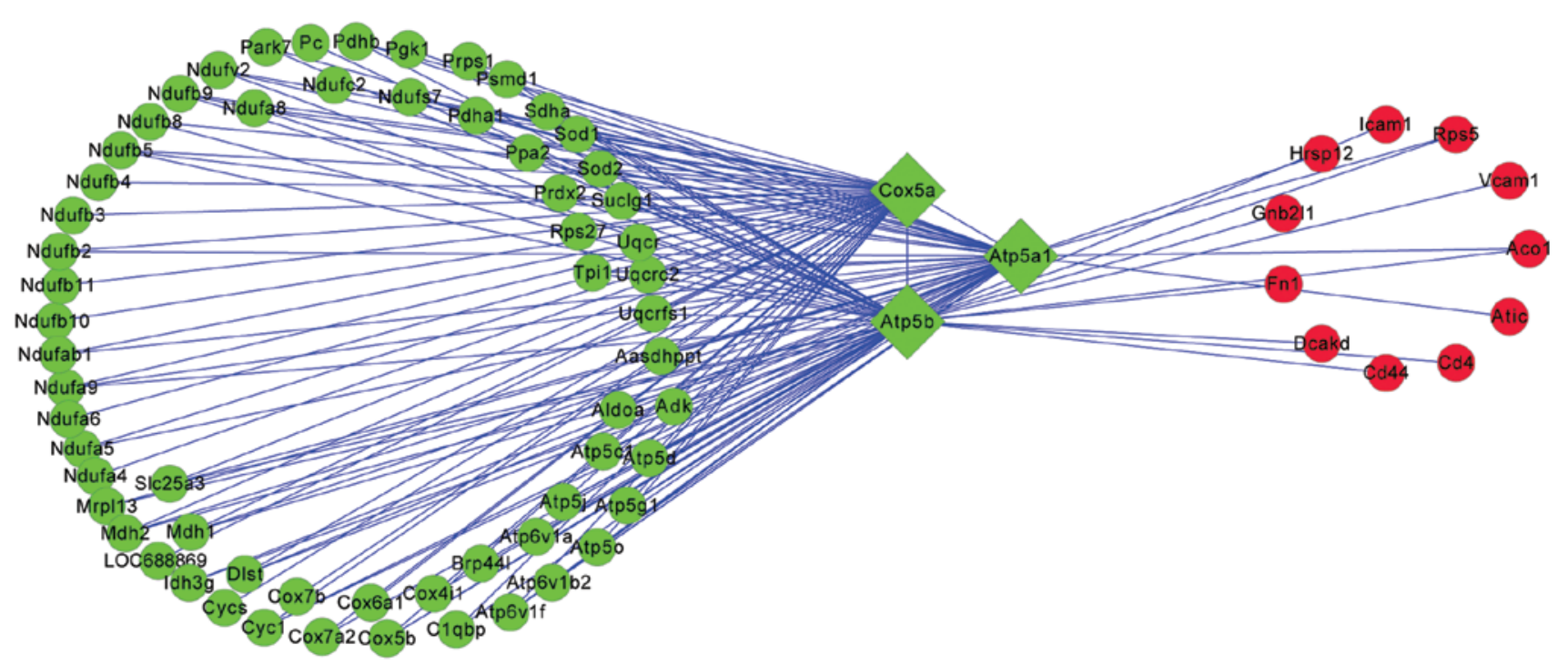

Figure 3. Interaction network of feature genes associated with disease 35 days after spinal cord injury. The rhombi indicate feature genes and circles indicate interacting genes. The green color indicates downregulated genes, while the red color indicates upregulated genes.

genes that may be associated with the disease at 35 days after the spinal cord injury are shown in Table IV.

As shown in Table IV, the protein domain in the coding areas of Cox5a belonged to the COX5A family, 13 sub-unit complex, EC: 1.9.3.1, which is the terminal oxidase in the mitochondrial electron transport chain (26). By contrast, the protein domain in the coding areas of Atp5a and Atp5b belong to the ATP synthase $\alpha$ and $\beta$ family, including ATP-synt_ab_N, ATP-synt_ab and ATP-synt_ab_C. The ATP synthase $\alpha / \beta$ family includes the ATP synthase $\alpha$ and $\beta$ subunits and ATP synthase, associated with flagella (27).

\section{Discussion}

In the present study, it was demonstrated that the three feature genes, Cox5a, Atp5al and Atp5b, in the injured spinal cord, were rapidly downregulated 35 days after the onset of injury, resulting in the destruction of the mitochondrial electron transport chain and membrane-bound enzyme complexes/ion transporters. These genes have been reported to be involved in the pathways of several types of neurological disease, including Huntington's disease, Parkinson's disease and Alzheimer's disease $(28,29)$. Since these processes are associated with the 
transportation of energy in biological bodies, changes to these processes 35 days after SCI may result in disruption in the transport of energy.

COX5A is a protein-coding gene. It is a multi-subunit enzyme complex, which couples the transfer of electrons from cytochrome $c$ to molecular oxygen and contributes to a proton electrochemical gradient across the inner mitochondrial membrane (26). Diseases associated with COX5A include acquired idiopathic sideroblastic anemia and cardioencephalomyopathy (30). Its associated super-pathways include the electron transport chain and metabolic pathways. GO annotations associated with this gene include electron carrier activity and cytochrome $c$ oxidase activity (31). This indicates that COX5A may be important in the regulation and assembly of the complex in the human mitochondrial respiratory chain enzyme, thus, affecting energy supply in SCI. A previous study revealed that COX5A is associated with the migration, invasion and prediction of distant metastasis (32). In the present study, COX5A was markedly downregulated in SCI, therefore, it was hypothesized that the downregulation of COX5A in SCI caused the interdiction of energy transportation, interrupting the metabolic process.

ATPases, or ATP synthases, are membrane-bound enzyme complexes/ion transporters, which combine ATP synthesis and/or hydrolysis with the transport of protons across a membrane. ATPases harness the energy from a proton gradient, using the flux of ions across the membrane via the ATPase proton channel, to drive the synthesis of ATP (33). Atp5a1 (34) and Atp5b (35) are also protein-coding genes. Super-pathways associated with the genes include the electron transport chain and adenosine ribonucleotides de novo biosynthesis. GO annotations associated with ATP5A1 include eukaryotic cell surface binding and ATPase activity (36), while those for ATP5B include transmembrane transporter activity and transporter activity $(37,38)$. Deregulated energy metabolism is a marker of malignant disease, which offers possible future targets for treatment (39). Polymorphism and association analysis has revealed that mutations in Atp5a1 and Atp5b genes may be potential markers of diseases associated with the destruction of energy transport (40). Atp5a1 and Atp5b, which are involved in energy transportation in mitochondria, may be critical genes and certain variations of these genes may lead to increased risk in SCI (40).

In addition, the results obtained from GO enrichment analysis of the PPI network in the present study demonstrated that most enriched GO terms of the DEGs in SCI were associated with mitochondria, including 'mitochondrial electron transport chain', 'mitochondrial membrane' and 'mitochondrial envelope'. This suggested that the majority of DEGs in SCI were associated with energy transportation and that the progression of SCI may be affected by the genes expressed differently in the tissue. Therefore, the 39 mutual genes in Huntington's disease, Parkinson's disease and Alzheimer's disease, which coordinate with genes in SCI, may assist in defining the origins of malignancies and offer promise for earlier diagnosis and improved treatment of SCI.

In conclusion, the results of the present study presented a comprehensive bioinformatics analysis of genes and pathways, which may be involved in the progression of SCI. A total of 929 DEGs were identified from GSE2599, and PPI networks were constructed using these DEGs. Furthermore, the Cox 5a, Atp5al and Atp5b genes, which were downregulated in SCI, were found to result in the destruction of the mitochondrial electron transport chain and membrane-bound enzyme complexes/ ion transporters, thus affecting the normal function of nerves. These genes can be identified as feature genes of SCI and assist in the early diagnosis and improved treatment of SCI.

\section{References}

1. Richards JS, Kewman DG, Pierce CA, Frank R and Elliott T: Spinal cord injury. Handbook of rehabilitation psychology: 11-27, 2000.

2. Karimi MT: Evidence-Based Evaluation of Physiological Effects of Standing and Walking in Individuals with Spinal Cord Injury. Iran J Med Sci 36: 242, 2011.

3. Mcdonald JW and Sadowsky C: Spinal-cord injury. The Lancet 359: 417-425, 2002.

4. Bareyre FM and Schwab ME: Inflammation, degeneration and regeneration in the injured spinal cord: insights from DNA microarrays. Trends Neurosci 26: 555-563, 2003.

5. Fraser A and Edmonds-Seal J: Spinal cord injuries. Anaesthesia 37: 1084-1098, 1982.

6. Xia T, Ni S, Li X, et al: Sustained delivery of dbcAMP by poly (propylene carbonate) micron fibers promotes axonal regenerative sprouting and functional recovery after spinal cord hemisection. Brain Res 1538: 41-50, 2013.

7. Hayashi M, Ueyama T, Nemoto K, Tamaki T and Senba E: Sequential mRNA expression for immediate early genes, cytokines, and neurotrophins in spinal cord injury. J Neurotrauma 17: 203-218, 2000.

8. Dergham P, Ellezam B, Essagian C, Avedissian H, Lubell WD, and Mckerracher L: Rho signaling pathway targeted to promote spinal cord repair. J Neurotrauma 22: 6570-6577, 2002.

9. Nakahara S, Yone K, Sakou T, et al: Induction of apoptosis signal regulating kinase 1 (ASK1) after spinal cord injury in rats: possible involvement of ASK1-JNK and-p38 pathways in neuronal apoptosis. J Neuropathol Exp Neurol 58: 442-450, 1999.

10. Aimone JB, Leasure JL, Perreau VM, Thallmair M: Spatial and temporal gene expression profiling of the contused rat spinal cord. Experimental neurology 189:204-221, 2004.

11. Troyanskaya O, Cantor M, Sherlock G, et al: Missing value estimation methods for DNA microarrays. Bioinformatics 17: 520-525, 2001

12. Fujita A, Sato JR, Rodrigues Lde O, Ferreira CE and Sogayar MC: Evaluating different methods of microarray data normalization. BMC Bioinformatics 7: 469, 2006.

13. Smyth GK: Limma: linear models for microarray data. In: Bioinformatics and Computational Biology Solutions using $\mathrm{R}$ and Bioconductor. Springer, New York: 397-420, 2005.

14. Benjamini Y and Hochberg Y: Controlling the false discovery rate: a practical and powerful approach to multiple testing. J R Stat Soc Series B Stat Methodol 57: 289-300, 1995.

15. Nam D and Kim SY: Gene-set approach for expression pattern analysis. Brief Bioinform 9: 189-197, 2008.

16. Allison DB, Cui X, Page GP and Sabripour M: Microarray data analysis: from disarray to consolidation and consensus. Nat Rev Genet 7: 55-65, 2006.

17. Huang Da Wei BTS and Lempicki RA: Systematic and integrative analysis of large gene lists using DAVID bioinformatics resources. Nat Protoc 4: 44-57, 2008.

18. Huang Dw SB and Lempicki Ra: Systematic and integrative analysis of large gene lists using DAVID Bioinformatics Resources. Nat Protoc 4: 44-57, 2009.

19. Hulsegge I, Kommadath A and Smits MA: Globaltest and GOEAST: two different approaches for Gene Ontology analysis. BMC Proc 3 Suppl 4: S10, 2009.

20. Huang Da W, Sherman BT and Lempicki RA: Systematic and integrative analysis of large gene lists using DAVID bioinformatics resources. Nat Protoc 4: 44-57, 2009.

21. Franceschini A, Szklarczyk D, Frankild S, et al: STRING v9. 1: protein-protein interaction networks, with increased coverage and integration. Nucleic Acids Res 41: D808-D815, 2013.

22. Shannon P, Markiel A, Ozier O, et al: Cytoscape: a software environment for integrated models of biomolecular interaction networks. Genome Res 13: 2498-2504, 2003.

23. Saito R, Smoot ME, Ono K, et al: A travel guide to Cytoscape plugins. Nat Methods 9: 1069-1076, 2012. 
24. Burge $C$ and Karlin S: Prediction of complete gene structures in human genomic DNA. J Mol Biol 268: 78-94, 1997.

25. Bateman A, Coin L, Durbin R, et al: The Pfam protein families database. Nucleic Acids Res 32: D138-D141, 2004.

26. Malström BG: Cytochrome c oxidase Structure and catalytic activity. Biochim Biophys Acta 549: 281-303, 1979.

27. Sauer K, Cullen M, Rickard A, Zeef L, Davies D and Gilbert P: Characterization of nutrient-induced dispersion in Pseudomonas aeruginosa PAO1 biofilm. J Bacteriol 186: 7312-7326, 2004.

28. Liang WS, Reiman EM, Valla J, et al: Alzheimer's disease is associated with reduced expression of energy metabolism genes in posterior cingulate neurons. Proc Natl Acad Sci 105: 4441-4446, 2008.

29. Emahazion T, Jobs M, Howell WM, Siegfried M, Wyöni P-I, Prince JA and Brookes AJ: Identification of 167 polymorphisms in 88 genes from candidate neurodegeneration pathways. Gene 238: 315-324, 1999.

30. Capaldi RA: Structure and function of cytochrome c oxidase. Annu Rev Biochem 59: 569-596, 1990.

31. Miller BR and Cumsky MG: An unusual mitochondrial import pathway for the precursor to yeast cytochrome c oxidase subunit Va. J Cell Biol 112: 833-841, 1991.

32. Chen W-L, Kuo K-T, Chou T-Y, et al: The role of cytochrome c oxidase subunit Va in non-small cell lung carcinoma cells: association with migration, invasion and prediction of distant metastasis. BMC cancer 12: 273, 2012.
33. Rappas M, Niwa $\mathrm{H}$ and Zhang X: Mechanisms of ATPases - a multi-disciplinary approach. Curr Protein and Pept Sci 5: 89-105, 2004.

34. DeKloet SR: Loss of the Gene for the Subunit of ATP Synthase (ATP5A1) from the W Chromosome in the African Grey Parrot (Psittacus erithacus). J Mol Evol 2, 2001.

35. Zheng S-Q, Li Y-X, Zhang Y, Li X and Tang H: MiR-101 regulates HSV-1 replication by targeting ATP5B. Antiviral Res 89: 219-226, 2011

36. Jonckheere AI, Renkema GH, Bras M, et al: A complex V ATP5A1 defect causes fatal neonatal mitochondrial encephalopathy. Brain 136: 1544-1554, 2013.

37. Doi K and Uetsuka K: Mechanisms of mycotoxin-induced neurotoxicity through oxidative stress-associated pathways. Int J Mol Sci 12: 5213-5237, 2011.

38. Sineshchekova OO, Kawate T, Vdovychenko OV and Sato TN: Protein-trap version 2.1: screening for expressed proteins in mammalian cells based on their localizations. BMC Cell Biol 5: 8, 2004.

39. Hjerpe E, Brage SE, Carlson J, et al: Metabolic markers GAPDH, PKM2, ATP5B and BEC-index in advanced serous ovarian cancer. BMC Clin Pathol 13: 30, 2013.

40. Gunawan A, Sahadevan S, Cinar MU, et al: Identification of the Novel Candidate Genes and Variants in Boar Liver Tissues with Divergent Skatole Levels Using RNA Deep Sequencing. PloS One 8: e72298, 2013. 